\title{
Clinical Results Comparing Transtibial Technique and Outside in Technique in Single Bundle Anterior Cruciate Ligament Reconstruction
}

\author{
Seung Suk Seo, $\mathrm{MD}^{1}$, Chang Wan Kim, $\mathrm{MD}^{2}$, Jeon Gyo Kim, $\mathrm{MD}^{2}$, and Sung Yub Jin, $\mathrm{MD}^{2}$ \\ ${ }^{1}$ Department of Orthopedic Surgery, Bumin Hospital, Busan; ${ }^{2}$ Department of Orthopedic Surgery, Inje University Busan Paik Hospital, Inje University College of \\ Medicine, Busan, Korea
}

\begin{abstract}
Purpose: To compare the clinical results of single-bundle anterior cruciate ligament (ACL) reconstruction using the conventional transtibial technique and the anatomical outside-in technique for femoral tunneling.

Materials and Methods: From 2007 to 2011, 89 patients who received ACL reconstruction were followed for $\geq 1$ year were enrolled in the study. The conventional transtibial technique was used in 41 patients and the outside-in technique, in 48 patients. Femoral tunnel angle measurement and threedimensional computed tomography (3D CT) were used for radiologic assessment of the location of femoral tunnel and Lysholm score and other tests were used for clinical assessment.

Results: Both techniques did not reveal statistical differences in the clinical assessment. However, in International Knee Documentation Committee subjective knee evaluation, the sum of two questionnaire items regarding instability showed a statistically significant difference ( $\mathrm{p}=0.01)$. In the pivot shift test, the anatomical outside-in technique showed outstanding rotational stability over the transtibial technique ( $\mathrm{p}=0.04)$. The mean femoral tunnel inclination in coronal plane were $69.2^{\circ}$ and $30.3^{\circ}$, respectively, for both techniques, and $21.6^{\circ}$ and $50.8^{\circ}$, respectively in sagittal plane, showing statistically significant differences on simple radiography $(\mathrm{p}=0.04,0.05)$. A 3D CT was performed in 17 patients with the conventional transtibial technique and 25 patients with the outside-in technique. Coefficients of variation were 0.33 and 0.13 , respectively, from dorsal border of the condyle and 0.67 and 0.24 , respectively, from the roof of intercondylar notch.

Conclusions: Femoral tunnels created with the outside-in technique have superior knee joint rotational stability compare to the transtibial technique. Therefore, the outside-in technique could be considered as a valuable technique in single-bundle ACL reconstruction.
\end{abstract}

Keywords: Knee, Anterior cruciate ligament, Reconstruction, Transtibial technique, Outside-in technique

\section{Introduction}

Anterior cruciate ligament (ACL) dysfunction leads to the progression of osteoarthritis and adjacent tissue damage caused by

Received October 22, 2012; Revised (1st) January 31, 2013;

(2nd) June 13, 2013; (3rd) July 16, 2013; Accepted July 17, 2013

Correspondence to: Chang Wan Kim, MD

Department of Orthopedic Surgery, Inje University Busan Paik Hospital, Inje University College of Medicine, 75 Bokji-ro, Busanjin-gu, Busan

614-735, Korea

Tel: +82-51-890-6129, Fax: +82-51-892-6619

E-mail: doctor-bluesea@hanmail.net

This is an Open Access article distributed under the terms of the Creative Commons Attribution Non-Commercial License (http://creativecommons.org/licenses/by-nc/3.0/) which permits unrestricted non-commercial use, distribution, and reproduction in any medium, provided the original work is properly cited. knee instability ${ }^{1)}$. The goal of ACL reconstruction is to restore the normal stability and kinetics of the knee joint. Factors that can influence the outcomes of an ACL reconstruction include patient selection, surgical technique, postoperative rehabilitation, and the extent of combined ligament injury. With regard to the surgical technique, femoral and tibial tunnel positioning, graft selection, tension to the graft, and graft fixation method are of particular importance $e^{2,3)}$. In spite of the constant improvement in surgical techniques, the presence of rotational instability and development of delayed osteoarthritis have still been the focus of concern in ACL reconstruction. Anatomical tunnel placement has been suggested as a strategy to reduce rotational instability and complications of ACL reconstruction, such as arthritis ${ }^{4}$. The advances in anatomy and biomechanics of the knee have shifted the concept of a proper femoral tunnel position from the is ometric point to the anatomic location ${ }^{5)}$. The anatomic position of a tibial tunnel is 
between the anteromedial (AM) and posterolateral (PL) bundles of the ACL or at the center of the tibial insertion site of the entire ACL. The anatomic position of a femoral tunnel is away from the posterior margin of the femoral ACL footprint by the same distance between the anterior margin of the ACL footprint and the center of the tibial tunnel when the knee is in $90^{\circ}$ flexion. The most common techniques for femoral tunnel placement are the transtibial technique, AM portal technique, and outside-in technique. Giron et al. ${ }^{6}$ reported that anatomic femoral tunnel placement was unattainable even with any modifications of the transtibial techniques. In addition, the AM portal technique resulted in creation of a relatively short femoral tunnel and had the risk of causing damage to the articular cartilage of the medial femur. The orientation of the femoral tunnel becomes more horizontal with the outside-in technique than with the transtibial technique. Some recent biomechanical studies have suggested that a more oblique femoral tunnel in the coronal plane is advantageous in preventing anterior translation and internal rotation of the tib$\mathrm{ia}^{7-9)}$. On the other hand, one of the drawbacks of the outside-in technique is the need for an additional incision. In this study, we compared the clinical and radiographic results of single-bundle ACL reconstructions using a conventional transtibial technique and an outside-in technique under the hypothesis that the latter that allows for anatomic reconstruction would produce more satisfying clinical results, rotational stability in particular.

\section{Materials and Methods}

\section{Patients}

Of the patients who had undergone a single-bundle ACL reconstruction between 2007 and 2011, 89 patients who were available for $\geq 1$-year follow-up were included in this study. The ACL reconstruction was performed using a conventional transtibial technique in 41 patients and an outside-in technique in 48 patients. The ACLs were reconstructed with tibialis tendon allografts in all patients. The transtibial technique was used for ACL reconstruction until August 2009 and the outside-in technique was employed thereafter. The mean age of the patients at the time of surgery was 31.02 years (range, 19 to 41 years). The mean follow-up period was 30 months (range, 13 to 57 months). The mechanism of injury was sports activities in 24 patients (58.5\%) in the transtibial group and in 29 patients (60.4\%) in the outside-in technique group. The combined injury was a meniscal tear and a medial collateral ligament injury in 8 and 2 patients, respectively, in the transtibial group and in 10 and 3 patients, respectively, in the outside-in technique group. Conservative
Table 1. Patients Demographics

\begin{tabular}{lccc}
\hline \multicolumn{1}{c}{ Variable } & Transtibial & Outside-in & p-value \\
\hline Sex (male:female) & $32: 9$ & $40: 8$ & 0.54 \\
Age (yr), mean \pm SD & $30.6 \pm 11.14$ & $32.4 \pm 13.3$ & 0.40 \\
Sport injury (\%) & $24(58.5)$ & $29(60.4)$ & 0.35 \\
Combined injury (case) & & & \\
$\quad$ Meniscus injury & 8 & 10 & 0.56 \\
MCL injury & 2 & 3 & 0.63 \\
\hline
\end{tabular}

MCL: medial collateral ligament.

treatment was performed for the stable meniscal tear that was defined as $\leq 3 \mathrm{~mm}$ tear of the peripheral rim in 3 patients. For the remaining meniscal tears, arthroscopic suture repair was carried out. Patients who underwent a concomitant surgery in ACL reconstruction for other combined injuries, such as a medial/lateral collateral ligament injury, posterior cruciate ligament injury, and PL structure injury, were excluded from the study. There was no statistically significant difference between the two groups regarding age, gender, and combined injury (Table 1).

\section{Conventional Transtibial Technique for Femoral Tunnel Placement}

ACL reconstruction using the conventional transtibial technique was performed in the order of tibial tunnel placement, femoral tunnel drilling, and graft insertion and interference screw fixation. After confirming the ACL tear with arthroscopy, a minimal notchplasty was performed to prevent graft impingement. The remnants of the torn ACL were debrided prior to reconstruction. A tibial dill guide (Arthrex Inc., Naples, FL, USA) set at an angle of $45^{\circ}-50^{\circ}$ was inserted through the AM portal and the center of the exit point of the guide was placed 1-2 mm posterior to the center of the tibial footprint. The entry point of the tibial tunnel was placed anterosuperior to the junction of the medial collateral ligament and pes anserinus and a guidewire oriented at $40^{\circ}-45^{\circ}$ with regard to the tibial axis was drilled. With the knee placed in approximately $90^{\circ}$ flexion, a femoral tunnel guide (Arthrex Inc.) was introduced through the tibial tunnel inside the joint. With the tip of the femoral guide aimed at the 10:30 or 1:30 position, a guidewire was positioned at the center and drilled. A graft was placed in the femoral tunnel, rotated by $120^{\circ}$, and fixated with Rigidfix (Mitek, Norwood, MA, USA). For graft fixation in the tibial tunnel, after confirming there was no impingement between the graft and the intercondylaar notch, interference screw fixation was performed with the knee in $30^{\circ}$ flexion and a $40 \mathrm{~N}$ tension applied to the graft using the SE Graft 
Tensioning System (Linvatec Co., Largo, FL, USA). Subsequently, after ligating the suture on the cortical screw, additional fixation was performed with a pike washer.

\section{Anatomic Femoral Tunnel Placement Using the Outside-in Technique}

Femoral tunnel drilling was performed in a retrograde fashion with the FlipCutter (Arthrex Inc.), taking care to match the tunnel diameter with that of the graft. Since the FlipCutter is available in various sizes with an increment of $1 \mathrm{~mm}$, the size of the FlipCutter was determined according to the diameter of the graft. With the knee in $90^{\circ}$ flexion, the native ACL position was examined by placing the tip of an arthroscopic probe on the proximal articular border of the femur: it located just below the over-thetop position and just above the superior margin of the cartilage of the lateral femoral condyle. The center of the ACL remnants that was located halfway between the posterior fossa of the lateral intercondylar ridge and the lateral bifurcate ridge was marked with a microfracture awl. Subsequently, the FlipCutter was inserted using the femoral guide that was oriented at $90^{\circ}-100^{\circ}$. With the FlipCutter, a 20 or $25 \mathrm{~mm}$ deep femoral socket that allows for the space for the flipped Endobutton Fixation Device (Smith \& Nephew, Andover, MA, USA) was created. As in the conventional transtibial technique, the entry point of a tibial tunnel was placed anterosuperior to the junction of the medial collateral ligament and pes anserinus, and a guidewire was drilled. After confirming there is no impingement between the graft and the intercondylaar notch with the knee in extension, the graft was fixed with an absorbable screw of a diameter equal to the tibial tunnel size under a $40 \mathrm{~N}$ tension applied by the SE Graft Tensioning System. After interference screw fixation using an absorbable screw that matches the diameter of the tibial tunnel, supplemental fixation was performed with a spiked washer following ligature of the suture around the $4.0 \mathrm{~mm}$ cortical screw.

\section{Clinical Assessment}

On the clinical assessment, two orthopedic surgeons evaluated range of motion (ROM), Lachman test grade, pivot shift test grade, International Knee Documentation Committee (IKDC) subjective knee score, Lysholm score, and Tegner activity level score at the last follow-up in the outpatient clinic. Ligament laxity was assessed using the KT1000 Arthrometer (Medmetric Co., San Diego, CA, USA).

\section{Radiographic Assessment}

The coronal inclination and sagittal inclination of the femo-
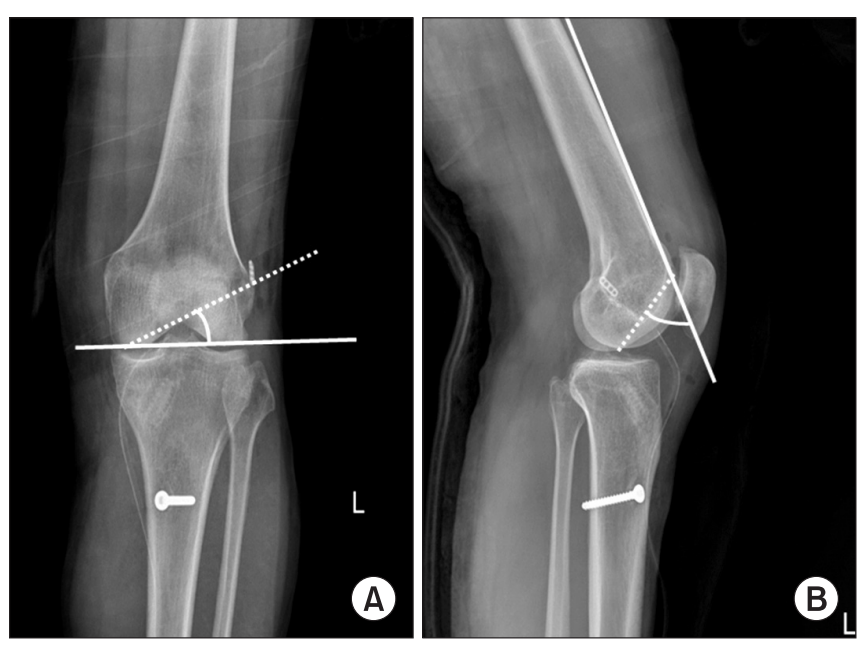

Fig. 1. (A) A line parallel to the axis of the femoral tunnel and the distal femoral condylar line were used to calculate the coronal inclination of the femoral tunnel. (B) Sagittal inclination of the femoral tunnel in relation to the posterior femoral cortex in lateral view.

ral tunnel were assessed for comparison of the two techniques. The coronal inclination of the femoral tunnel was measured as the angle between the long axis of the femoral tunnel and a line drawn along the intercondylar articular surface of the femur on the anteroposterior radiograph. The sagittal inclination was measured as the angle between the long axis of the femoral tunnel and a line tangent to the posterior femoral cortex on the lateral radiograph (Fig. 1).

\section{Three-Dimensional Computed Tomography (3D CT) \\ Assessment}

A CT scan with a slice thickness of $1.25 \mathrm{~mm}$ was performed between the 2 nd and 4 th postoperative weeks. The data from the CT scan was reconstructed into 3D images using the Aquarius Net Viewer (V4.4.6.85, TeraRecon, San Mateo, CA, USA). The location of the femoral tunnel on the postoperative $3 \mathrm{D}$ reconstructed CT scan image was assessed using the quadrant method described by Bernard and Hertel ${ }^{10)}$; a grid that was formed by a line parallel to the Blumensaat's line that corresponds to the roof of the intercondylar notch and another line tangent to the posterior wall of the lateral femoral condyle was superimposed onto the image (Fig. 2).

\section{Statistical Analysis}

Statistical analysis of clinical data was performed using SPSS ver. 11.0 (SPSS Inc., Chicago, IL, USA). Fisher's exact test was used to analyze the IKDC subjective knee score and the Lachman test grade. The Mann-Whitney test was used to compare differences 

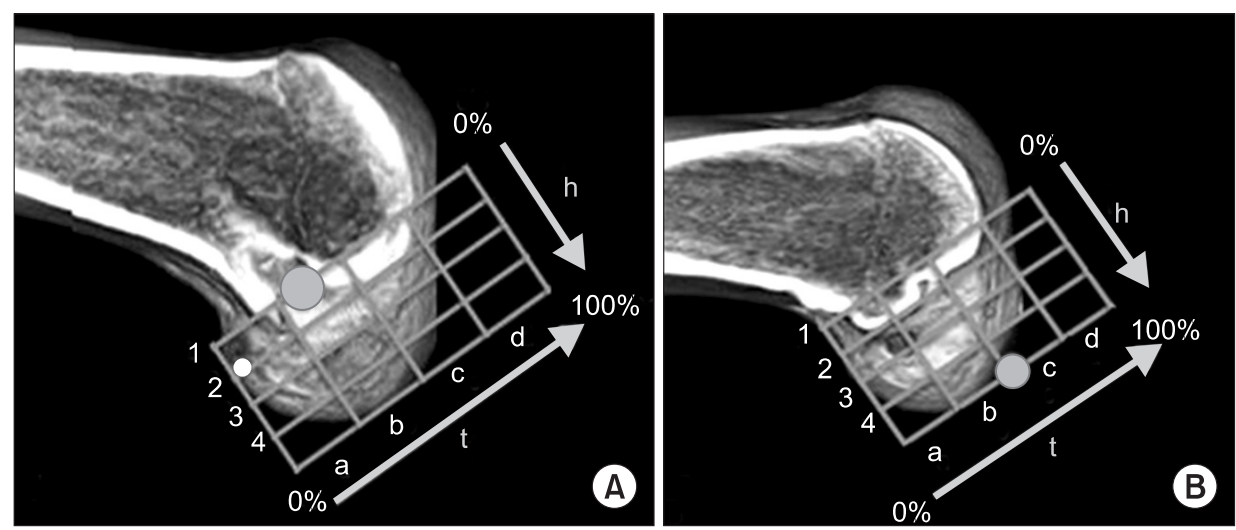

Fig. 2. The location of the femoral tunnel aperture centers were established within a $4 \times 4$ grid, which was oriented along the most anterior edge of the notch roof. (A) Transtibial technique. (B) Outside-in technique. h: line perpendicular to the Blumensaat's line, t: line parallel to the Blumensaat's line.

in the Lysholm grade and Tegner activity level score. A p-value of the KT1000 Arthrometer test was calculated using a paired t-test. The pivot shift test grade was evaluated using the chi-square test. The patients in the outside-in technique group were subdivided into three groups for comparison: those with a standard coronal inclination of the femur $\left(30^{\circ}-59^{\circ}\right)$ were assigned into group II $(n=18)$ and those with less or more than the standard inclination were classified into group I $\left(0^{\circ}-29^{\circ}, \mathrm{n}=23\right)$ or group III $\left(\geq 60^{\circ}\right.$, $\mathrm{n}=7$ ), respectively. The Mann-Whitney $\mathrm{U}$ test was used to evaluate subgroup differences. A $\mathrm{p}<0.05$ was considered statistically significant for all statistical analyses.

The intraobserver reliability of the radiographic assessment performed by the two observers was assessed using the intraclass correlation coefficients (ICC). A unpaired t-test was conducted for statistical comparison of the two techniques with a significance level set at $\mathrm{p} \leq 0.05$.

The intraobserver reliability of the postoperative 3D CT image assessment done by the two orthopedic surgeons was evaluated using ICC.

\section{Results}

The mean Lysholm score was $91.4 \pm 4.8$ in the transtibial group and $93.2 \pm 12.6$ in the outside-in group. The mean ROM was $135.6 \pm 12.6$ in the transtibial group and $139.4 \pm 8.8$ in the outsidein group. The Lachman test results in the transtibial group and the outside-in group were grade 0 in 26 patients and 32 patients, respectively, grade I in 13 patients and 16 patients, respectively, and grade II in 2 patients and none, respectively, and grade III was not observed in both groups. The mean Tegner activity level score was $4.9 \pm 5.4$ in the transtibial group and $5.1 \pm 6.9$ in the outside-in group. The mean ligament laxity assessed using the KT-1000 arthrometer in the transtibial group and the outside-in group was $2.82 \pm 2.5$ and $2.64 \pm 2.1$, respectively: $\leq 3 \mathrm{~mm}$ ligament
Table 2. Comparison of Pivot Shift Test between Transtibial and Outside-in Technique

\begin{tabular}{lcc}
\hline \multicolumn{1}{c}{ Grade } & Transtibial & Outside-in \\
\hline 0 (absence) & 18 & 36 \\
1 (glide) & 19 & 18 \\
2 (clunk) & 3 & 4 \\
3 (gross) & 1 & 0 \\
\hline
\end{tabular}

Fisher's exact test. $\mathrm{p}<0.042$.

laxity was observed in 30 patients and 35 patients, respectively; 3-5 mm, in 8 patients and 12 patients, respectively; and $\geq 5 \mathrm{~mm}$, in 3 patients and 1 patient, respectively. No statistically significant difference between the two techniques was observed in the clinical assessment ( $\mathrm{p}=0.56,0.19,0.07,0.12$, and 0.23 ). The IKDC subjective knee score in the transtibial group and the outside-in group was $85-100$ points in 28 patients and 33 patients, respectively, 70-84 points in 11 patients and 14 patients, respectively, and 55-69 points in 2 patients and 1 patient, respectively, and 0-54 points was not observed in both groups, showing no significant difference between the two techniques. However, the mean score of the two items for joint instability assessment (item 7 and 8, 4 points each) in the IKDC subjective knee evaluation form was $5.44 \pm 1.21$ in the transtibial group and $6.67 \pm 1.13$ in the outside-in group, showing significant difference $(p<0.01)$. The pivot shift test was performed with the hip fully abducted and the tibial band relaxed to improve accuracy of the test. While applying a valgus stress on the proximal tibia with one hand to cause anterior subluxation of the tibia, the examiner grasped the heel of the involved leg with the opposite hand and internally rotated the tibia as the knee is extended. The pivot shift test grade in the transtibial group and the outside-in group was grade 0 in 18 patients and 36 patients, respectively, grade I in 19 patients and 18 patients, respectively, grade II in 3 patients and 4 patients, respec- 
tively, and grade III in 1 patient and none, respectively, showing significant difference between the two techniques $(\mathrm{p}<0.04)$. In particular, the pivot shift test grade was significantly low in the outside-in group I compared to the outside-in group III $(\mathrm{p}<0.05)$ (Table 2, 3).

The mean coronal inclination of the femoral tunnel on the plain radiograph was $69.2^{\circ} \pm 10.2^{\circ}$ in the transtibial group and $30.3^{\circ} \pm 7.5^{\circ}$ in the outside-in group and the mean sagittal inclination was $21.6^{\circ} \pm 7.8^{\circ}$ in the transtibial group and $50.8^{\circ} \pm 4.5^{\circ}$ in the outside-in group, showing statistically significant difference between the two techniques ( $\mathrm{p}=0.04,0.03$ ). The ICC for intraobserver reliability was excellent: it was 0.92 for the coronal inclination measurement and 0.94 for the sagittal inclination measurement.

The $3 \mathrm{D}$ reconstructed CT images obtained from 17 patients in the transtibial group and 25 patients in the outside-in group who were available for the 3D CT scan showed that the center of the femoral tunnel was located $32.3 \% \pm 10.8 \%$ and $27.5 \% \pm 3.7 \%$, respectively, from the posterior articular surface and $18.8 \% \pm 12.6 \%$ and $36.4 \% \pm 4.8 \%$, respectively, from the roof of the intercondylar notch. The coefficient of variation $(\mathrm{CV})$ used to assess distri-

Table 3. Comparison of Pivot Shift Test between Transtibial and Subgroups of Outside-in Technique

\begin{tabular}{lcccc}
\hline \multicolumn{1}{c}{ Grade } & Transtibial & $\begin{array}{c}\text { Outside-in I } \\
\left(<30^{\circ}\right)\end{array}$ & $\begin{array}{c}\text { Outside-in II } \\
\left(30^{\circ}-60^{\circ}\right)\end{array}$ & $\begin{array}{c}\text { Outside-in III } \\
\left(>60^{\circ}\right)\end{array}$ \\
\hline 0 (absence) & 18 & 11 & 80 & \\
1 (glide) & 19 & 9 & 9 & 0 \\
2 (clunk) & 3 & 3 & 1 & 6 \\
3 (gross) & 1 & 0 & 0 & 1 \\
\hline
\end{tabular}

Coronal inclination at anteroposterior radiograph. Mann-Whitney U test. $\mathrm{p}<0.048$. bution of the femoral tunnel relative to the posterior articular surface was 0.33 in the transtibial group and 0.13 in the outsidein group and that relative to the roof of the intercondylar notch was 0.67 in the transtibial group and 0.24 in the outside-in group. Thus, the outside-in technique exhibited higher reproducibility in terms of the femoral tunnel placement than the transitbial technique (Fig. 3). The ICC for intraobserver reliability was excellent for both measurements of the relative position of the femoral tunnel from the posterior articular surface (0.93) and from the femoral intercondylar notch (0.91).

\section{Discussion}

Factors that influence the outcome of an ACL reconstruction include patient selection, surgical technique, postoperative rehabilitation, and concomitant ligament injury. Tibial and femoral tunnel positioning and graft type, tension, and fixation method should also be taken into consideration prior to surgery. Improper bone tunnel positioning can result in abnormal graft tension, reduced ROM, postoperative instability, recurrence of the ACL injury, and a graft tear.

A femoral tunnel can be created using a transtibial technique, AM portal technique, or outside-in technique. The transtibial technique requires relatively short operative time and exhibits high reproducibility. One of the major advantages of the technique is that the femoral tunnel that is created through the tibial tunnel can be positioned at the optimum isometric point ${ }^{11,12)}$. In the long-term, the use of a transitbial technique in ACL reconstruction results in decreases in the Lachman test grade and anterior tibial dislocation assessed with arthrometer, but slippage and axial instability are often observed ${ }^{7}$.

The AM approach provides greater rotational stability because of the transverse orientation of the femoral tunnel than the trans-

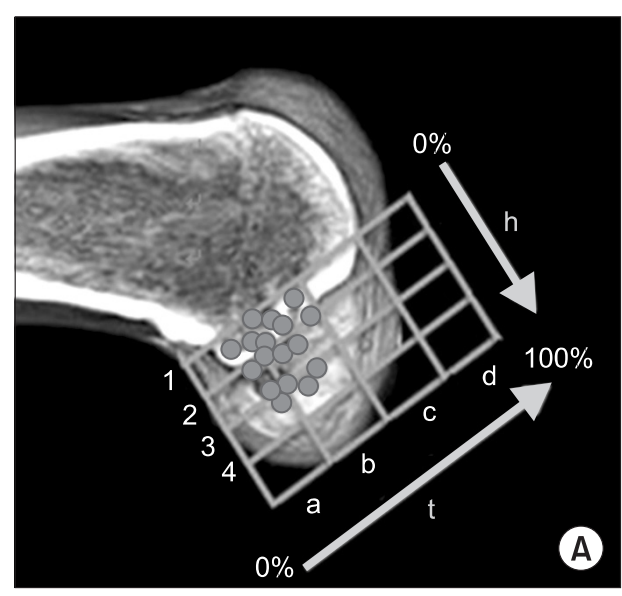

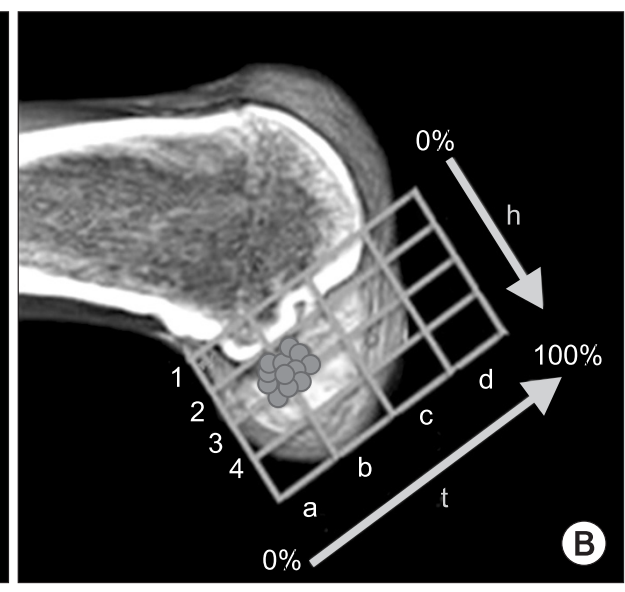

B
Fig. 3. Each distribution of femoral tunnels in (A) transtibial technique and (B) outsidein technique. h: line perpendicular to the Blumensaat's line, t: line parallel to the Blumensaat's line. 
tibial technique. However, there is no sufficient number of midand long-term follow-up studies to prove such a benefit, there is a risk of creating a short femoral tunnel, compromising the posterior tunnel wall, and increasing the need for revision ${ }^{13)}$.

Lubowitz and Konicek $^{14)}$ reported that the outside-in technique could be performed through a small incision that is approximately the size of an arthroscope without additional lateral incision and useful for preventing excessively short femoral tunneling as opposed to the AM technique.

In our study, there was no significant difference between the two techniques in the clinical outcome, except for the rotational instability. The IKDC subjective knee scores for two items related to knee joint stability and pivot shift test grade were higher after ACL reconstruction using the outside-in technique. The pivot shift test has been used as the standard method to evaluate rotational instability of the knee in many recent studies ${ }^{15,16)}$. In addition, based on recent advances in biomechanical research, the pivot shift test has been considered as an indicator that can quantify rotational instability as well as anterior translation ${ }^{17)}$.

In congruent with the aforementioned studies, the coronal inclination was approximately $30^{\circ}$ more vertical when the femoral tunnel was created with the transtibial technique in this study. Vertical orientation of the femoral tunnel in the coronal plane has been associated with restoration of the anterior stability but postoperative rotational instability as well ${ }^{18-20)}$. A variety of recent biomechanical studies have demonstrated there is a positive correlation between the horizontal femoral tunnel position in the coronal plane and rotational stability, especially stability for internal rotation of the tibia ${ }^{4,9,11,12)}$. The sagittal inclination was significantly more horizontal in parallel with the femoral cortex when the femoral tunnel was created using the outside-in technique. This means a greater divergence between the femoral tunnel and the posterior cortex and thus greater preservation of the thickness of the posterior wall of the femoral tunnel, which reduces damage to the posterior cortex during interference screw fixation ${ }^{21)}$. In a study by Hantes et al. ${ }^{22)}$ the ACL graft was in a more oblique direction in the sagittal plane compared to the orientation of the normal ACL, which was necessitated due to more oblique tibial tunneling for obtaining a longer tibial tunnel.

However, horizontal femoral tunnel orientation on the coronal plane results in a steep angle between the graft and the femoral tunnel, which may increase the risk of graft wear ${ }^{23,24)}$. In an attempt to reduce the wear-related damage, Graf et al. ${ }^{23)}$ suggested femoral tunnel chamfering.

A statistically significant difference between the two techniques was found in the femoral tunnel position on the $3 \mathrm{D}$ recon- structed CT scan assessed using the quadrant method. Several recent studies have shown that the position of the femoral tunnel created using the outside-in technique is close to the optimal position suggested by the quadrant method, which implies that the outside-in technique is more effective for anatomical femoral tunnel placement ${ }^{25-27)}$. On the comparison of the $\mathrm{CV}$ that is the standard deviation divided by the mean, the CVs for femoral tunnel position relative to the two references on the $3 \mathrm{D}$ CT scan were smaller for the outside-in technique, showing that the outside in technique has higher reproducibility for femoral tunnel positioning and that it is difficult to obtain consistency in femoral tunnel placement with the transtibial technique.

The limitations of this study include that long-term follow-up results could not be provided due to the relatively short follow-up after the ACL reconstruction using the outside-in technique, the follow-up period was not identical between the two groups, and the 3D CT scan was not performed in all patients.

The significance of this study is that it shows the outside-in technique that creates a femoral tunnel with a more transverse coronal inclination at the anatomical position provides greater improvement in rotational stability than does the transtibial technique.

\section{Conclusions}

The outside-in technique exhibited higher reproducibility compared to the transtibial technique and was more effective for providing rotational stability than the nonanatomical ACL reconstruction technique.

\section{Conflict of Interest}

No potential conflict of interest relevant to this article was reported.

\section{References}

1. Feller J. Anterior cruciate ligament rupture: is osteoarthritis inevitable? Br J Sports Med. 2004;38:383-4.

2. Bealle D, Johnson DL. Technical pitfalls of anterior cruciate ligament surgery. Clin Sports Med. 1999;18:831-45.

3. Graf B, Uhr F. Complications of intra-articular anterior cruciate reconstruction. Clin Sports Med. 1988;7:835-48.

4. Loh JC, Fukuda Y, Tsuda E, Steadman RJ, Fu FH, Woo SL. Knee stability and graft function following anterior cruciate ligament reconstruction: comparison between 11 oclock and 
10 o'clock femoral tunnel placement. 2002 Richard O'Connor Award paper. Arthroscopy. 2003;19:297-304.

5. Snyder GM, Johnson DL. Anatomic graft placement in ACL surgery: plain radiographs are all we need. Orthopedics. 2011;34:116-8.

6. Giron F, Cuomo P, Edwards A, Bull AM, Amis AA, Aglietti P. Double-bundle "anatomic" anterior cruciate ligament reconstruction: a cadaveric study of tunnel positioning with a transtibial technique. Arthroscopy. 2007;23:7-13.

7. Markolf KL, Hame S, Hunter DM, Oakes DA, Zoric B, Gause P, Finerman GA. Effects of femoral tunnel placement on knee laxity and forces in an anterior cruciate ligament graft. J Orthop Res. 2002;20:1016-24.

8. Ristanis S, Stergiou N, Siarava E, Ntoulia A, Mitsionis G, Georgoulis AD. Effect of femoral tunnel placement for reconstruction of the anterior cruciate ligament on tibial rotation. J Bone Joint Surg Am. 2009;91:2151-8.

9. Scopp JM, Jasper LE, Belkoff SM, Moorman CT 3rd. The effect of oblique femoral tunnel placement on rotational constraint of the knee reconstructed using patellar tendon autografts. Arthroscopy. 2004;20:294-9.

10. Bernard M, Hertel P. Intraoperative and postoperative insertion control of anterior cruciate ligament-plasty: a radiologic measuring method (quadrant method). Unfallchirurg. 1996;99:332-40.

11. Howell SM, Gittins ME, Gottlieb JE, Traina SM, Zoellner TM. The relationship between the angle of the tibial tunnel in the coronal plane and loss of flexion and anterior laxity after anterior cruciate ligament reconstruction. Am J Sports Med. 2001;29:567-74.

12. Simmons R, Howell SM, Hull ML. Effect of the angle of the femoral and tibial tunnels in the coronal plane and incremental excision of the posterior cruciate ligament on tension of an anterior cruciate ligament graft: an in vitro study. J Bone Joint Surg Am. 2003;85:1018-29.

13. Bedi A, Raphael B, Maderazo A, Pavlov H, Williams RJ 3rd. Transtibial versus anteromedial portal drilling for anterior cruciate ligament reconstruction: a cadaveric study of femoral tunnel length and obliquity. Arthroscopy. 2010;26:34250.

14. Lubowitz JH, Konicek J. Anterior cruciate ligament femoral tunnel length: cadaveric analysis comparing anteromedial portal versus outside-in technique. Arthroscopy. 2010;26:1357-62.

15. Meredick RB, Vance KJ, Appleby D, Lubowitz JH. Outcome of single-bundle versus double-bundle reconstruction of the anterior cruciate ligament: a meta-analysis. Am J Sports Med. 2008;36:1414-21.

16. Yagi M, Kuroda R, Nagamune K, Yoshiya S, Kurosaka M. Double-bundle ACL reconstruction can improve rotational stability. Clin Orthop Relat Res. 2007;454:100-7.

17. Lane CG, Warren R, Pearle AD. The pivot shift. J Am Acad Orthop Surg. 2008;16:679-88.

18. Jepsen CF, Lundberg-Jensen AK, Faunoe P. Does the position of the femoral tunnel affect the laxity or clinical outcome of the anterior cruciate ligament-reconstructed knee? A clinical, prospective, randomized, double-blind study. Arthroscopy. 2007;23:1326-33.

19. Johnson DL, Swenson TM, Irrgang JJ, Fu FH, Harner CD. Revision anterior cruciate ligament surgery: experience from Pittsburgh. Clin Orthop Relat Res. 1996;(325):100-9.

20. Kaseta MK, DeFrate LE, Charnock BL, Sullivan RT, Garrett WE Jr. Reconstruction technique affects femoral tunnel placement in ACL reconstruction. Clin Orthop Relat Res. 2008;466:1467-74.

21. Albuquerque RF, Amatuzzi MM, Pacheco AP, Angelini FJ, Campos Jr O. Positioning of the femoral tunnel for arthroscopic reconstruction of the anterior cruciate ligament: comparative study of 2 techniques. Clinics (Sao Paulo). 2007;62:613-8.

22. Hantes ME, Zachos VC, Liantsis A, Venouziou A, Karantanas $\mathrm{AH}$, Malizos KN. Differences in graft orientation using the transtibial and anteromedial portal technique in anterior cruciate ligament reconstruction: a magnetic resonance imaging study. Knee Surg Sports Traumatol Arthrosc. 2009;17:880-6.

23. Graf BK, Henry J, Rothenberg M, Vanderby R. Anterior cruciate ligament reconstruction with patellar tendon: an ex vivo study of wear-related damage and failure at the femoral tunnel. Am J Sports Med. 1994;22:131-5.

24. Kim KT, Sohn SK, Chang YS, Chung IK. Comparision of trans-tibial and anteromedial portal approach in femoral tunneling of anterior cruciate ligament reconstruction. J Korean Arthrosc Soc. 2004;8:75-81.

25. Colombet P, Robinson J, Christel P, Franceschi JP, Djian P, Bellier G, Sbihi A. Morphology of anterior cruciate ligament attachments for anatomic reconstruction: a cadaveric dissection and radiographic study. Arthroscopy. 2006;22:984-92.

26. Zantop T, Wellmann M, Fu FH, Petersen W. Tunnel positioning of anteromedial and posterolateral bundles in anatomic anterior cruciate ligament reconstruction: anatomic and radiographic findings. Am J Sports Med. 2008;36:65-72. 


\section{Seo et al. Transtibial and Outside in Technique in ACL Reconstruction}

27. Forsythe B, Kopf S, Wong AK, Martins CA, Anderst W, Tashman S, Fu FH. The location of femoral and tibial tunnels in anatomic double-bundle anterior cruciate ligament reconstruction analyzed by three-dimensional computed tomography models. J Bone Joint Surg Am. 2010;92:1418-26. 